\title{
Phonetic Functions in The Glossary of Persian Arabic Expressions
}

\author{
Hadeel Raad Tahsin Al Darraji \\ College of Education for Women, University of Anbar, Iraq \\ dr.hadeelrt@gmail.com
}

\begin{abstract}
:
Because of the kinship in Semitic languages of the prominent place in the cultural and social system of the owners of these languages and the presence of a semi- clear between these vocabulary in most Semitic languages has in flounced to address some of these vocabulary through this research study the level of voice and the impact of Arabic in Persian in order to stand causes of the variables suffered by the ease and clarify their changes and recognize the formal voice harmony and the role played by these voices in the system communicate.
\end{abstract}

Keywords: Vocalizations; Phonemes; Semitic Languages; Similarities; Differences.

Crossref doi https://doi.org/10.51345/.v31i1.329.g146 


\section{REFERENCES:}

Al -Istrabadi: Muhammad bin Al-Hassan Radhi Al-Din Al-Istrabadi, Sharh Al-Radhi on Al-Kafia, Corrected and Commented by Yusef Hassan Omar, Professor at the Faculty of Arabic Language and Islamic Studies, Qar Younis University, 1398 AH - 1978 AD.

Al-Qaisi: Makki Al-Qaisi Abu Muhammad Makki bin Abi Talib (d. 437 AH), the problem of the parsing of the Qur'an, by :Hatem Al-Damen, Freedom House Printing, Baghdad, 1975.

Al-Ansari: Muhammad bin Makram bin Ali Abu Al-Fadl Jamal Al-Din Ibn Manzoor Al-Ansari (d. 711 AH), Lisan Al-Arab, Dar Al-Nawadir Publishing (Beirut - Lebanon) 1985.

Al-Asadi: Abu al-Baqaa Ya`sh ibn Ali ibn Ya`sh al-Asadi (643 AH), Explanation of the Kingship in Al-Tasrif, Achievement of Fakhr al-Din Qibawa, The Arab Library of Aleppo, I 1, 1973.

Al-Askari: Abu Ahmad Al-Hassan bin Abdullah bin Saeed Al-Askari (d. 382 AH), investigation by Abdul Aziz Ahmed, Mustafa Al-Babi Al-Halabi Library, 1st edition, 1963.

Al-Askari: Abu Ahmad Al-Hassan bin Abdullah bin Saeed Al-Askari (d. 383 AH), investigation by Abdul Aziz Ahmed, 1st edition, Mustafa Al-Halabi and Sons Press - Egypt, 1963.

Al-Asqalani: Ahmad bin Ali bin Muhammad bin Hajar al-Asqalani (d. 773 AH, (a picnic looking at elucidating the elite of thought, investigation by Abdullah bin Dhaif Allah al-Rahili, 1st edition, 2001.

Al-Attiyah: Khalil Ibrahim Al-Attiyah, Voice Research by the Arabs, i 1, Baghdad, Dar Al-Jahiz Publications 1976.

Al-Azdi: Abu Bakr Muhammad bin Al-Hassan bin Dureid Al-Azdi (d. 321 AH), The Population of Language, a symbolic investigation by Munir Baalbaki, Dar Al-Alam for Millions - Beirut, 1st edition, 1987 $\mathrm{AD}$.

Al-Bakoush: Doctor Al-Bakoush, The Arabic Drainage, The Investigation of Saleh Al-Qarmadi, Mu'nes Publishing House, Arab Press, 1992.

Al-Farahidi: Hebron Ahmed bin Ahmed Al-Farahidi (d. 170 AH), Al-Ain, investigation by Dr.Mehdi Makhzoumi and d .Ibrahim Al-Samarrai, Baghdad, 1980.

Al-Halawani, Muhammad Khair al-Halawani, the clear in the science of exchange, Dar Al-Mamoun Heritage, Damascus, 1407 AH - 1987 AD.

Al-Hamouz: Abdel-Fattah Al-Homouz, The Spatial Heart Phenomenon in Arabic - Its Causes, Evidence, and Interpretation of its Types, Dar Ammar (Amman - Jordan), 1st edition, 1986.

Al-Harawi: Muhammad bin Ahmed bin Al-Azhari Al-Herawi Abu Mansour (d. 370 AH) Refining the language, investigation by Muhammad Awad Marib, Arab Heritage Revival House - Beirut, 1st edition, 2001 $\mathrm{AD}$.

Al-Jawhary: Ismail bin Hammad (d. 393 AH), Al-Sahah, "The Language of Linguistics" and "Sahih AlArabiya”, by Ahmed Abdel Ghafour Attar, Dar Al-Alam for Millions, Beirut, Fourth Edition, 1407 AH-1987 CE.

Al-Jurjani: Abu al-Hasan al-Jurjani (Ali bin Muhammad bin Ali, known as al-Sharif 740 AH - 816 AH), definitions, Muhammad Asaad Press, (D-T.(

Al-Mursi: Abu Al-Hassan Ali bin Ismail bin Saydah Al-Mursi (d. 458 AH) Al-Musaas, investigation by Khalil Ibrahim Jaffal, publisher of the Arab Heritage Revival House - Beirut, 1st edition, 1417 AH-1996 AD.

Al-Suyuti: Abu Bakr Jalal Al-Din Al-Suyuti (d. 911 AH), Al-Mizhar in Language Sciences and its Types, explained by Muhammad Jad Al-Mawla and two others, published by Dar Al-Jeel, Beirut, (D.T.(

Al-Zamakhshari: Abu al-Qasim Mahmoud bin Amr bin Ahmad al-Zamakhshari, Jarallah (d. 538 AH), the superior in the hadith and hadeeth effect, investigation (Ali Muhammad al-Bajawi) and (Muhammad Abu al-Fadl Ibrahim), Dar al-Maarefa - Lebanon, 2nd floor.

Al-Zubaidi: Abu Bakr al-Zubaidi, the tune of the common people, investigation d .Ramadan Abdel Tawab, Cairo 1964. 
Al-Zubaidi: Muhammad ibn Muhammad ibn Abd al-Razzaq al-Husayni, Abu al-Faid, called Mortada al-Zubaidi (d. 1205), the crown of the bride from the jewels of the dictionary, a group of investigators, Dar alHidaya (numbering of the book is OK for printing.(

Anis: Dr. Ibrahim Anis, Linguistic Voices, 5th Edition, The Egyptian Anglo Library, 1975 AD.

Anis: Dr .Ibrahim Anis, in Arabic Dialects, Egyptian Anglo Press, Cairo 1965.

Ed Sher: The Archbishop Ed Sher (d. 1333 AH): A Dictionary of Persian Arabic Words, Catholics Press 1958, Beirut.

El-Desouky: Sheikh Mohamed Ali El-Desouky, Abstract of Refining Vernacular Words, Cairo, 1924.

Genie: Abu Al-Fath Othman bin Jani (d. 392 AH), Phonological and Phonological Studies, Dr. Hussam AlNuaimi, Dar Al-Tale'ah, Beirut 1980.

Genie: Abu Fateh Othman bin Jani (d. 392), The Secret of Making Arabic, Dar Al-Qalam - Damascus, 1st edition, 1985 Hassan Hindawi.

Good: Dr .Ali Khleif Hassan, Curriculum of the audio lesson for Arabs, Dar Al-Kutub Al-Alami, Beirut Lebanon, 1st edition, 2011.

Hasan: Al-Sayyid Muhammad ibn al-Sayyid Hasan (d. 869 AH), Al-Ramooh Al-Sahah, investigation by Ali Abdul Karim Al-Rudaini, published by Usama Dar - Damascus, 2nd edition, 1986 AD.

Ibn Jani: Abu al-Fath bin Uthman (d. 392) Characteristics, investigation by Muhammad Ali al-Najjar, Cairo, 2nd edition, 1955.

Ibn Yaish: Mowaffaq al-Din Yaish bin Ali bin Yaish (d. 643 AH), Sharh Al-Mufsal, Al-Muniriya Press, Egypt, (D.T.(

Litwab, Dr. Ramadan Abdel-Tawab, Introduction to Linguistics and Curricula of Linguistic Research, AlMadani Press, Cairo, 1st edition, 1,1403 AH -1982 CE.

Matlabi: Ghalib Fadel Matlabi, in Language Voices, Republic of Iraq - Ministry of Culture and Information, House of Cultural Affairs 1984.

Omar: Ahmed Mokhtar Omar, The Study of Linguistic Voice, First Edition, (Cairo - Egypt) Books World 1997.

Omar: Dr .Ahmed Mukhtar Omar, The Study of Linguistic Voice (Dr. i), The World of Books in Cairo (1396 AH - 1976 AD. (

Repentance: Dr .Ramadan Abdel Tawab, General Tune and Linguistic Development, 1 st edition, Cairo, 1967 AD.

Repentance: Dr .Ramadan Abdel-Tawab, chapters in philology, 3rd floor, Al-Khanji Library, Egypt, 1408 AH 1987 CE.

Shaheen: Dr .Abdel-Sabour Shaheen, the effect of readings on Arabic voices and grammar, Al-Khanji Library Cairo, 1st edition, 1978 AD.

Sibawayh Al-Kitab: Amr bin Othman bin Qanbar Al-Harthy, loyalty and known as Sibawayh (d. 180 AH). The book, investigation by Abdul Salam Haroun - Beirut 1403 AH - 19 83AD.

Tawab: Ramadan Abdel Tawab, linguistic development, manifestations, reasoning and laws.

The cooler: Abu al-Abbas Muhammad ibn Yazid ibn Abd al-Akbar (d. 285) Al-Muqtazb, investigation by Muhammad Abd al-Khaliq Azimah, Cairo, 1963, part 1.

The Prices: Mahmoud Al-Sifran, Linguistics, 2nd floor, Dar Al-Fikr Al-Arabi, Cairo, 1997.

What for mereng: Bertil Ma Lamberg, phonology, translation and study d .Abdel-Sabour Shaheen, 1st Floor, Cairo - Egypt, Youth Library 1984.

Zamakhshari as the basis of rhetoric: Abu al-Qasim Mahmoud bin Amr bin Ahmed Al-Zamakhshari Jarallah (d. $538 \mathrm{AH})$ The basis of rhetoric, investigation by Ahmed Basil Oyoun Al-Aswad, Dar Al-Kutub AlAlami, Beirut - 1998.

Daz and j: Reinhart - Peter Anne Dozy (deceased 1300 e) supplement dictionaries Arabic, moved into Arabic and commented upon by Mohammed Salim Al Nuaimi, and Jamal Al - Khayat, the publication of the Ministry of Culture and Information, Republic of Iraq, i 1, from 1979-2000 m. 


\section{الظو اهر الصوتية الوظائفية في معجم الألفاظ الفارسية المعربة \\ أ.م.د. هديل رعد تحسين الدراجي \\ كلية التربية للبنات، جامعة الأنبار، العراق \\ dr.hadeelrt@gmail.com}

ملخص البـحث

نظراً لما تحتله ألفاظ القرابة في اللغات السامية من مكانة بارزة في النظام الثقافي والاجتماعي لأصحاب هذه اللغات ، ووجود شبه واضح بين هذه الألفاظ في معظم اللغات السامية ، فقد أثرت أن أعالج بعض هذه الألفاظ من خلال هذا البحث ، بدراسة المستوى الصوتي وتأثير العربية بالفارسية ، بهدف الوقوف على وجوه التشابه ، والاختلاف ، والاصول المشتر كة بينها . ثم تحديد اسباب المتغيرات التي لحقت بها نحو السهولة والتيسير ، فنحاول التخلص من الاصوات العسيرة النطق بأصوات تتطلب جهداً عضلياً ، وتوضيح معالمها التغيرية واقرار شكلية الانسجام الصوتي ، والدور الذي تلعبه هذه الاصوات في نظام

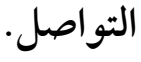

الكلمات المفتاحية : الألفاظ، الصوتية، اللغات السامية، التشابه، الاختلاف.

Crossref doi https://doi.org/10.51345/.v31i1.329.g146 


\section{المقدمة}

أهم ما ركز عليه علماؤنا القدامى هو (الدراسات الصوتية) لما به من علاقة وثيقة بالنحو والصرف والمعجم و التفسير ، فإن بحثنا عن الدراسات الصوتية واللغوية والمعجمية والتي بدأت هذه الدراسات تستمد مناهجها من علم الأصوات العام ، وتأثير مناهج هذه العلوم على الدراسات الصوتية في اللغات الأخرى ، فهذا البحث يبين فكرة ربط اللغة بالتراث القديم بشكل عام ، والتراث الصوتي بشكل خاص ، وكيف أثرت بأفكارها ومصطلحاتها ومفاهيمها ومضامينها على اللغات الأخرى ، وهذا ما يمكن أن تستتجه من خلال القر اءة المتخصصة لما يكمن ورآه هذه العلوم من غايات و اهداف . ومن أجل تأكيد هذه الفكرة أرى أن أحصر القول على الدراسات الصوتية على ضوء الابحاث الصوتية الحديثة ، ويأتي هذا البحث ليعرف مسألة الصوتيات وعلاقتها بالانسان المعرفية والبحث في كيفية استثمارها نظرياً وعلمياً

فقد تناولت في هذا البحث مقدمة تحدثت فيها عن الموضوع وأسباب اختياري له وأهمية هذه الدراسة ، ومن ثم قسمت دراسة البحث على خمة محاور ، فالمحور الاول درست فيه ظاهرة القلب ، والمحور الثاني لظاهرت المماثلة الصوتية ، والمحور الثالث تناولت فيه المخالفة الصوتية ، أما المحور الرابع بينت فيه المعاقبة الصوتية ، والمحور الخامس : تناولت فيه ظاهرت التحريف والتصحيف باعتبارها دراسة صوتية ، ومن ثم عقدت الدراسة بخاتمة ذكرت فيها ما توصلت إليه من نتائج في هذا البحث .

\section{المحور الاول: القلب المكاني}

القلب في اللغة يقال : " قلب الشيء قلباً ، حوّله عن وجهه ، وكلامّهّوقلوب ، وقلب رداءه وقلبه لوجهه : كبّه ، وقلبه ظهر اَلِبَطن ، وقلب البيطار قوائم الدابة : رفعها ينظر إليها " (1) أما اصطلاحاً : فيعرب بأنه " تقديم بعض حروف الكلمة على بعض و أكثر ما ينفق في المعتل والمهموز . (2) 
وبين ابن جني بقوله : " إن أصل القلب في الحروف إنها هو فيها تقارب منها ، وذلك : الدال و الطاء ، والتاء،

$$
\text { والذال ، والظاءء ، والثاء....... وغير ذلك مما تدانت مخارجه " (3) }
$$

وذكر السيوطي : بخصوص ظاهرة القلب : القلب الصحيح عند البصريين مثل شاكي السلاح وشائك ، أما

ما يسميه الكوفيون : القلب نحو : جنوب وجبذ ، فليس بقلب عند البصريين ، وإنما هما لغتان . (4) وذلك " إن الصوت لا يمكن أن ينقلب إلى صوت آخر بعيد عنه في المخرج جداً ، فلا ينقلب صوت من أصوات الشفة أو الاسـنان مثلا إلى صوت آخر مـن أصوات الحلق وكذلك العكس " (5) ومن خلال ما سبق نعلل الظاهرة الصوتية : هو تدافع الحروف على اللسان فيولد الخطأ في غخارجها نتيجة السرعة في النطق • ويبدو أن اللغات السامية عرفت القلب المكاني وحتى اللغات اللاتينية ـ مما يدل على قدم هذه الظاهرة وتأصلها في اللغات السامية . ومثال ذلك ما ورد في معجم الالفاظ الفارسية

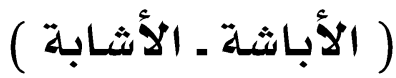

تعني الجماعة ، تقول جاءت أُباشة من الناس ، و ( الأباشة ) من الاخلاط و الرعاع ، و ( بَاش ) القوم اختلطوا فضجوا ، كل ذلك مأخوذ مـن أباش الفارسية وهي جماعة مـن كل أشكال الناس . (6) ونجد مثل هذه الظاهرة في هجة بأهلة أدرى القبائل العربية القديمة هذا اللون فذكر ابن فارس : " عُلط " : إنما ذلك مقوب والاصل " عُطل " وهي المرأة التي لا حلي ها والقياس واحد . (7) و ( البارزد مقلوب البازرد والاصل بارزو وهو دواء يعرف بالقنة ) (8) وكذلك (الجُباجب والجبَجَاب) بمعنى الكثير من الماء وهو مشتق من جاً بجاب وأصل معناه هنا وهناك ماء) 
ونجد شيوع هذا القلب وجدله متنفسا فسيحا وسط القبائل العربية البدوية ، وذلك لسرعة النطق وتدافع الحروف على اللسان والخطاً في اخراجها ، وعقد ابن دريد بابا سماه ( باب الحروف التي قلبت ) وذكر فيه طائفة من الالفاظ التي قلبت في كلام العرب كقوهم : ( ما أطيبه وما أيطبه ) (10) والذي يبدو أن هذا التباين يمثل العلاقة بين اللغات السامية واللغة الأم ، فيظهر هذا التجاذب بين خصائصها لأنها نمط غير ثابت .

ونجد ايضاً ظاهرة القلب المكاني تحدث بين الاصوات في السلسلة الكلامية في قوهم ( مرزاب ومزراب ) بتقدم الراء المهملة وتأخيرها ، وأصل الكلمة ميِز أي بَول ومن آب أي ماء، وتعني القناة يجري فيها الماء. (11) ومن خلال ما سبق تبين أكثر أنواع التدخل شيوعاً هي ( الاصوات ) إذ أن اللغة بتعريف ابن جني ـ أصوات يعبر بها كل قوم عن اغر اضهم (12). فالاصو ات تشكل اللغة ، وهذا النوع من الالتقاء بين اللغتين يشكل مهارة لغوية تعرف ( الأداء اللغوي ) ، وهذا النظام تحكمه مجموعة من التطورات كتوفير الجها واراحة النفس وإرضاء لرغبة جامحة في جانب من جوانب التفسير وفي نهاية المطاف يبحثون من الطريقة التي تؤدي ما يريدون وهو ( الانجاز اللغوي ) . (- موي

\section{المحور الثاني: المماثلة الصوتيـة}

المحاثلة تحدث عندما تتقارب اصو ات الكلمة بعضها مع بعض ، فتغير مخارج بعض الاصوات أو صفاتها لكي تتفق في المخرج أو في الصفة مع الأصوات الاخرى ، فيحدث ذلك النوع من التوافق والانسجام بين الاصوات المتنافرة في المخارج أو في الصفات ، ذلك أن أصوات اللغة تختلف فيها بينها في المخارج والشدة و الرخاوة والجهر والهمس وما إلى ذلك ، فكل صوت يحاول أن يجذب الآخر إلى ناحيته وييعله يتماثل معه في صفاته كلها أو في بعضها . (13) وهي من أبرز ظواهر العربية الفصحى (14)، تعود جذورها إلى الخليل ابن أحمد الفراهيدي ( 175 هـ ) الذي رأى أنه إذا تجاور صوتان أحدهما مجهور والآخر مهموس تفاعلا وتجاذبا حتى يتحدا في الصفة ويكونا بجهرين 
معاً أو مهموسين معاً (15)، فالماثلة تحول الفونيات المتخالفة إلى متماثلة إما تماثل كلي أو جزئي (16) ولكي

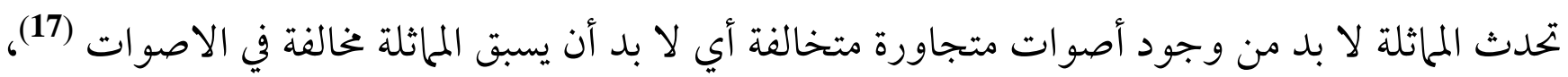
ويبقى الهدف وراء ذلك التأثير تحقيق التشابه أو التماثل بغية التقارب في الصفة والمخرج اقتصادا في الجهد العضلي المبذول (18) ومثال ما ورد في الالفاظ الفارسية المعربة :

\section{1 ـ التأثير المقبل الكلي ٍِِ حالة الاتصال ومن أمثلته:}

تتأثر تاء الافتعال دائما بالدال أو بالطاء قبلها فتقلب دالاً أو طاءٌ مثل ( الطَبرَدارج - بَرداج ) بمعنى حامل

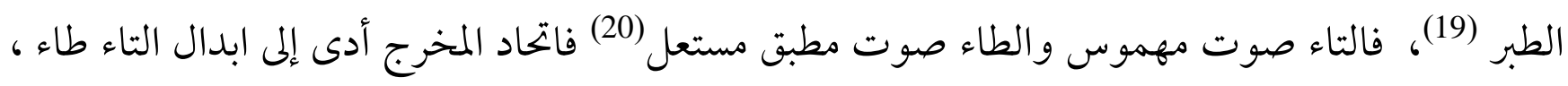

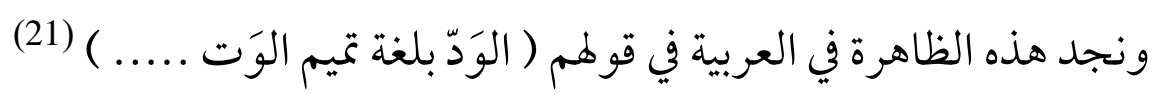

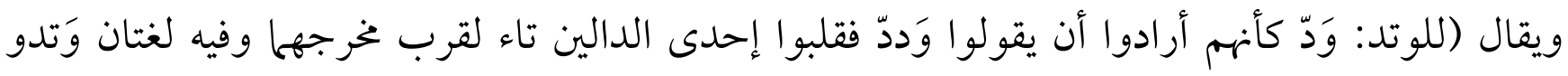
وتد).

وكثير ما ورد التهاثل في الالفاظ الفارسية منها ( طَبَاشير ـ تَباشير ) بمعنى دواء يكون في جوف القنا الهندي (23) وقوهم ( طابق - تابه ) فُسر بظرف يطبخ به وهي المقلاة (24) فئل

\section{2 ـ التأثير المقبل الكلي يِّ حالة الانفصال:}

وهنا تتحول الضمة إلى كسرة المرافقة لضمير النصب والجر الغائب المفرد المذكر بها قبلها كسرة طويلة أو

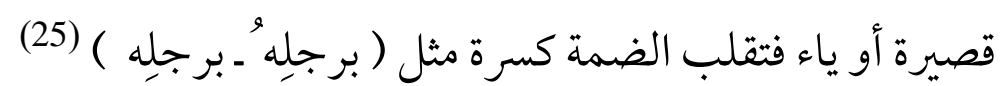

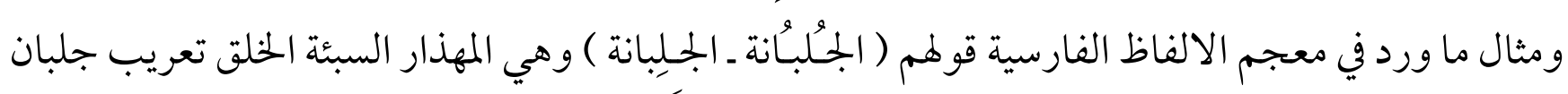

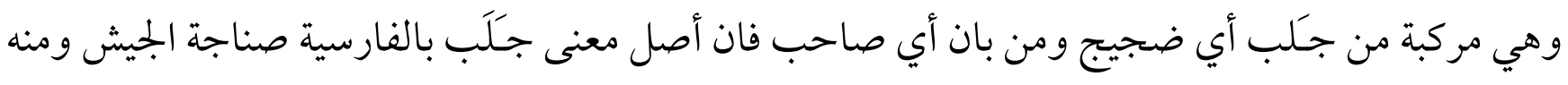
الضجيج والصياح · (26) 
ولتعزيز وجهة نظر هذه أورد ابراهيم أنيس صنفين بارزين في اللهجات العربية القديمة ، وهما القبائل البدوية

وتمثلها أسد ، ولهة الحضر تمثلها قبائل الحجاز ، وذكر أن الفتح لغة الحجاز والامالة لغة تميم (27) لذا اختيرت الكسرة للدلالة على المعنى الاقوى وهو ما يسد به من عوز وهذا يتطلب جهداً، لذا جاء اللفظ بالكسر

\section{3ـ التأثير المقبل الجزئي فِ حالة الاتصال:}

تتأثر تاء بالصاد أو بالضاد أو بالزاي فتقلب طاء في الحالتين الأوليتين ، ودالا في الحالة الثالثة مثل : ازتجر -

$$
\begin{aligned}
& \text { ازدجر ، واصتبغ ـ اصطبغ (28) - ماصن } \\
& \text { ومثال ما ورد في معجم الالفاظ الفارسية قولهم : } \\
& \text { ( الرطي - رتي ) بمعنى الاحمق وهي مأخوذة من أرت العريان والفقير (29). }
\end{aligned}
$$

وهذا التأثير الايجابي في النظام الادائي ، فالطاء حرف قوي متمكن والتاء مهموسة فحدث التهاثل ليقوى الكلام ، وهو ما ورد في قولمم ( الأستم ـ الاسطم ) بمعنى مجتمع البحر ومعناها بالفارسية الغدير والبحيرة

$$
\text { و.جع المياه (30) }
$$

ومثال الحالة الثالثة جاء قولهم في (الزّزداق ـ الرُستاق) بمعنى السو اد والقرى تعريب روستا ومنه الار رامي(31). وحصل هذا التأثير لأن خخرج الزاي ما بين الثنايا وطرف اللسان(32) والسين طرف اللسان وفويق الثنايا السفلى (33) حرف غير مستعل أي حروف الاستفال(34). وهذا تقارب ما بين الفارسية والعربية يعود إلى الافتراض اللغوي لتواصل الشعوب القاطنة في فارس والجزيرة العربية ادت إلى التقارب اللغوي .

\section{4 ـ التأثير المقبل الجزئي ِِ حالة الانفصال:}

تتأثر السين المهموسة بالر اء المجهورة قبلها فتقلب غلى نظيرها المجهور مثل ( مهراس ومهراز )(35). ومثال ما ورد في معجم الالفاظ الفارسية قولهم : 
( الصقر - الزقر ) بمعنى كل طائر يصيد من البراة والشواهن (36) وكذلك في ( الشُرم ـ الصُرم ) بمعنى

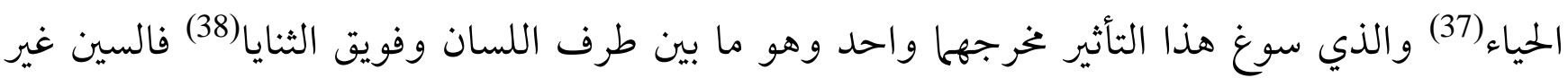

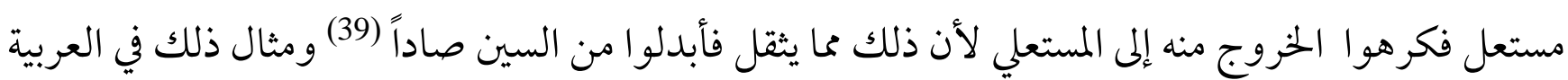

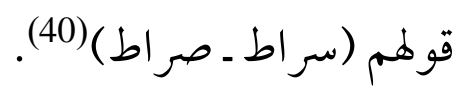
وكذلك تتاثر الضاد بالراء لأن الراء صوت ذو قيمة تفخيمية ونجد هذا في قولمم ( الرَوضة ـ ريز ) بمعنى

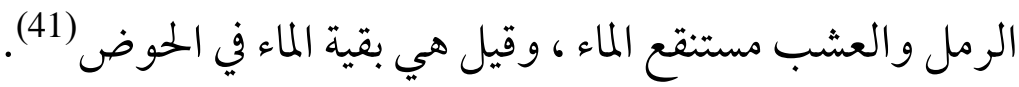

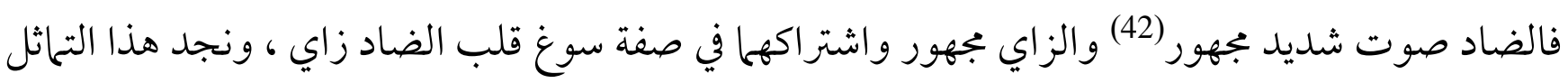
في اللغات السامية وقد فرضته البيئة الصوتية.

\section{5 ـ التأثير المدبر الجزئي ٍِِ حالة الانفصال :}

التقاء الصاد والراء في الكلمة تقلب زاياً مثل زراط في صراط ، وذكر اللخمي أن الناس كانوا في الاندلس والمغرب في القرن السادس الهجري في ( سرداب ـزرداب ) (43). ومثال ذلك في معجم الالفاظ الفارسية قوهم:

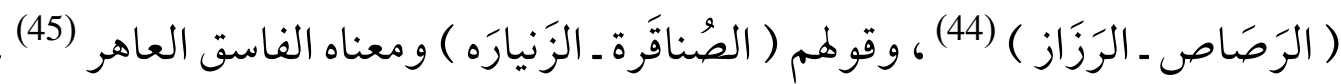
والذي سوغ قلب الحرف بين هذه الاصوات هو الاتفاق في المخرج فهي أصوات أسلية ؛ لأن مبدأها من أسلة اللسان ، وهو مستدق طرفه عند الخليل (46)، وهو ما بين طرف اللسان وفويق الثنايا(47).

\section{المحور الثالث: المخالفة الصوتية}

المخالفة ضد المماثلة ، وذلك أن يتحول أحسد الصوتين المتماثلين في الكلمة إلى صوت مغاير (48) فهي ن حقيقة أمرها تعديل في الصوت الموجود بتأثير صوت مجاور لكنه تعديل عكسي يؤدي إلى زيادة مدى الخلاف بين

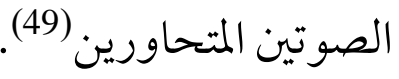


وذكرها القدماء ومنهم الفراهيدي الذي شبه اجتماع المثلين (بمشي المقيد ، لأنه يرفع رجله ، ويضعها في مو ضعها ، أو قريب منه ؛ لأن القيد يمنعه عن الانبعاث وامتداد الخطوة . (50) أما المحدثون يقول الدكتور أحمد مختار عمر : " إن المخالفة ينظر إليها على أنها تهدف إلى تيسير جانب الدلالة عن طريق المخالفة بين الاصوات ولا تلقي بالا إلى العامل النطقي الذي قد يتأثر نتيجة تباعد أو تخالف

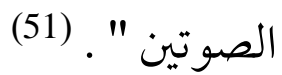
وعبر عنها بتسميات متعددة : كراهية التضعيف(52)، كراهية التشديد(53)، تكرار الأمثال(54) كراهية اجتماع المثلين أو المتجانسين (55)، والتخالف(56) وبذلك اتخذ علم|ء الدراسات الصوتية انها الوضع الأمثل اللازم لإعادة الخلافات بين الصوات ، وهو أمر ضروري لتحقيق حالة التوازن . فظاهرت المخالفة شائعة في اللغات السامية والعربية كلمة ( شمس ) والمعروف أن ( الشين ) في السامية الأم قلبت في العربية ( سينا ) ومقتضى ذلك تصير في العربية ( سمس ) غير أن المخالفة بين السينين أدت إلى قلب الاولى شينا" (57)

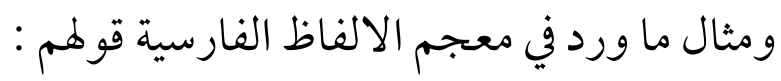
( الشَلجَم ـ و السَلجَم ) نبت يعرف باللفت ، وهي تعريب شملخ أو شلغم الفارسية (58) فالاول صوت أسلي والثاني شجري صوتان متقاربان مخرجاً ومتفقان صفة لذلك قلبت الشين سيناً . وكذلك قولهم ( الأرغيس ـ الأرغيش ) فُّر بقشر أصل شجر يصبغ به (59). وكذلك قولهم (الأصطَو انة ـ والأسطو انة) بمعنى قو ائم الدابة معرب أستون ومنه التركي والكردي يستون (60) فبدلت الصاد سيناً، و الذي سوغ ابدالها بين هذه الأصوات هو الاتفاق في المخرج ، فهي اصوات أسلية ـ لأن مبدأها من أسلة اللسان (61) كذلك وردت المخالفة في العربية قولمم ( قيراط ودينار ) بدلا من ( قّر اط ـ دّنار ) فيقول المبرد فالاصل : دّنار وقّر اط فابدلت الياء للكسرة فلم) فرقت بين المتضاعفين رجع الأصل فقلت ( دنانير وقراريط ) (62) 
و ومثال ما ورد في معجم الالفاظ الفارسية قولهم :

( الإبزِيّم ـ الابزام ) وتعني حلقة لما لسان تكون في السرج وغيره (63)

إبزام ابدلت الياء الف لتفريق المتضاعفين ، فأصبحت ابزام ؛ لأن الالف والياء من حيز واحد ، فهي عند الخليل هو ائية جوفية ، لأنها تخرج من الجوف (64)وهي متفقة في صفة الجهر والتوسط بين الشدة والرخاوة(65) وفي ضوء المخالفة عدت بعض الالفاظ العامية مثل ( لخبط ) التي يجدث فيها القلب من الفعل ( خلط )(66) وقد ذكر لها الاصمعي أمثلة كثيرة منها ( الزحاليف ـ الزحاليق ) وهي تزلج الصبيان فوق الطين ، مأخوذة من الفعل ( زحسّف ) كما أن الثانية مأخوذة من الفعل زحلق (67) ومثال ما ورد في معجم الالفاظ الفارسية قولهم : ( التوّز ـ التُوس ) وتعني الطبيعة والاصل قريبان لفظاً ومعنى من توُش ومعناه الجمسم القدرة (68)، و (الجِبز الجِبسب) وتفسر بالغليظ والبخيل واللئيم(69) فالمخالفة الصوتية ناتجة من اجتماع حرفين من جنس واحد فقلبت الزاي سين ، لأنها الاقرب في النطق ، وتنطق من أسلة اللسان ، وهي اسنانية لثوية كما ذكرها المحدثون(70)

$$
\text { فيقولون ( الرُداق ـ و الرستاق ) وتعني السوداء والقرى تعريب روستا(71) }
$$

كذلك وردت عن المخالفة الصوتية قلبهم الكاف قاف في لفظ ( الكافور ـ القافور ) تعريب كفرى وهو وعاء (72) (1) (1) فالقاف والكاف من خخرج واحد فها) لهويان مبدأهما من اللهاة عند الخليل (73) وعند سيبويه ( من أقصى اللسان وما فوقه من الحنك الاعلى )(74) فهي عندهم من حروف أقصى الحنك (75) متفقان في صفة الشدة (76) فوجدنا أن سبب المخالفة من الناحية الصوتية أن الصوتين متماثلين يحتاجان الى جهد عضلي في النطق في الكلمة الواحدة ، ولتيسير هذا المجهور يقلب أحد الصوتين صوتاً آخر. 


\section{المحور الرابع: المعاقبة الصوتية}

المعاقبة لغة: عاقب بين الشيئين إذا جاء بأحدهما مرة وبالاخر أخرى (77) ظاهرة ابدالية اختصت بين صوتين (الو او والياء) سببها دخول اللهجات القديمة في العربية الفصحى (78)، والمسوغ لحدوث المعاقبة هو القرابة الصوتية بين ( الواو والياء ) فعند الخليل صوتان هو ائيان من حيز واحد وهو الجوف (79) وعند سيبويه خرجها الشفتين(80) فكلاهما يشتركان في صفة الجهر(81)، يتصفان بالشدة عند سيبويه(82) والتوسط بين الشدة والرخاوة عند من جاء بعده (83). اصطلاحا: هو دخول الياء على الواو ، و العكس من غير علة تصريفية(84) ومثال ما ورد في معجم الالفاظ الفارسية المعربة قولهم :

( الدَريزَين ـ الدّرابزون ) وتعني قوائم مصفوفة تعمل من خشب أو حديد تحاط بها السلالم وغيرها، وهي مركبة من دراي باب ومن بزين أي تخت (85). كذلك قوهم ( السنطور ـ السنطير )، وفسرت الآت الطرب (86) و( الشولم ـ الشيلم ) وتعني الزوان (87)، و(الضَطر . الضوطر ) بمعنى من يدخل السوق بلا رأس مال فيحتال للكسب (88). وقد ورد في العربية مثل هذا التعاقب في قوهم (صواغ وصياغ ) في لغة أهل الحجاز ، تعاقب الواو ياءً(89). ومنهم من نطق الياء واواًمثل قبيلة فزارة ، يقولون ( كسايان ـ كساوان ). ونلاحظ أن علم)ء اللغة لم يحرصوا على وضع قوانين ومقاييس كالتي وضعها النحويون، وإنها اكتفوا برصد هذه الظاهرة ، وهذا ما أشار إليه سيبويه (90).

\section{المعاقبة بين الحركات}

لا يستطيع احد أن ينكر دور الحركة في اللغة على اختلاف مستوياتها ( صوتية صرفية نحوية دلالية ) وما أقرته الدراسات الصوتية الحديثة من أن الصوائت هي علامات الاعراب والاكثر وضوحاً في السمع ، وقد 
اكتسبت هذه الصفة في كونها أصوات بجهورة (91) والذي سوغ وقوع الابدال بينها هو تشابه الحركات في المخرج واتفاقها في بعض الصفات فالحركات كلها بجهورة رخوة (92).

\section{1 ـ التعاقب بين الكسر والفتح:}

ومثال ما ورد في معجم الالفاظ الفارسية المعربة قولهم : (الرَودند ـ الريوند) وهي اصل شجرة في الصين . وقيل اصل الرمان الهندي . فارسيته راوند (93). وهذا دليل الانسجام الصوتي الذي يمثل ظاهرة من ظواهر التطور في حركات الكلمة ، فالكلمة تشتمل على حركات متباينة تميل في تطورها إلى التوافق والانسجام بين الحركات(94). وهذا التداخل بين اللغات السامية يشكل أهم ملامح تأثير اللغة الأم في اللغات الاخرى ، وإذا انتقلنا إلى تأثير اللغة العربية نفسها يمكن القول إن ما تتميز به العربية من خصائص وميزات جمالية بكثرة التشابه بين أصواتها وصورها المكتوبة بينها وبين الفارسية .

\section{2 ـ تعاقب الكسر ـ فتح:}

جاء في معجم الالفاظ الفارسية المعربة قولهم : ( الجحسَاد ـ الجَسَّاد ) وهو الزعفران مشتق من ( الجَسَد ) (95) ويمكن أن نلاحظ هـذا التباين في الحركات عند القبائل العربية فمن سهات أهل الحجاز الكسر (96)، وذكر ابن جني أن العرب تفرق بين المعنيين بتغير حركة الحرف في بنية الكلمة ، ويختارون صوت الحركة الأقوى للمعنى الاقوى و الحركة الاضعف للمعنى الاضعف (97). إذن عملية التناوب بين الصوائت تعود إلى العلاقات الصوتية وهي المواضع النطقية متقاربة وصفتها واحدة .

3 ـ تعاقب الكسر ـ الضهم :

جاء في معجم الالفاظ قولمم ( الجِحنبار ـ الجُحُنبار ) 
وهو الرجل الضخم العظيم الخلق أو العظيم الجوف ، ويطلق بنوع خصوصي على الفيل والخصان ، وهو مركب من كُوة أي جبل ومن يـيحَار أي جثة (98). ولعل مثل هذه الدراسة لا تؤدي إلى اقرار نظرية شاملة أو تعقيد مسألة معينة إذ انها تؤدي لدلالة واحدة ، وهذا يظهر جلياً في المعنى الدلالي الذي منحته الصوائب لهذه الكلمة ، وهذا خالف في اللغة العربية إذ أن تناوب الصوائب الثلاثة في المثلثات اللغوية ، يمنح كل صائت منها للكلمة معنى ختلف عن المعاني التي تمنحها الصوائت الاخرى ويذلك تتحكم عملية التناةب بين الصوائت بدلالة اللفظية

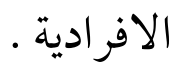

\section{4 ـ تعاقب الفتح ـ الضهم: \\ جاء قولهم : ( الجُردَبَان ـ الجُردُبان )} وهو الذي يضع شماله على شيء يكون الخوان لئلا يتناوله غيره فإذا فرغ القوم من الطعام يأكله ، وهو مأخوذ من ( كرُده ) ومعناه حافظ الرغيف (99).

ولعل سبب التعاقب ميلهم للفتح دون الضم ، أن الفتحة أخف من الضمة لان تجويف الفم يكون معها أكثر اتساعاً في قاع الفم (100).

\section{المحور الخامس : التصحيف والتحريف}

التصحيف : هو أن يقرأ الشيء بخلاف كاتبه وعلى غير ما اصطلح عليه في تسميته (101)، ولم يفرق القدماء بين التصحيف والتحريف كما ورد عند العسكري في كتابه ( شرح ما يقع فيه التصحيف والتحريف ) فأراد بالتحريف التصحيف وإبدال كلمة بأخرى (102). فالتصحيف هو ما كان فيه تغير في النقط ، أما التحريف هو ما كان فيه تغير في الشكل (103). ومن امثلة التحريف في معجم الالفاظ الفارسية المعربة قوهم : 
( الإنو ـ يم ) ويعني نصف الليل أو ما يقاربه و معناه النصف وهو (nema) بالسانسكريتية (104)، وهو ما أشار

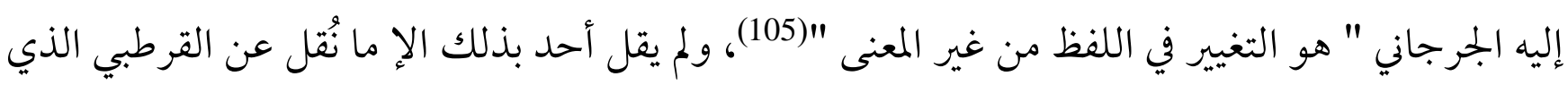

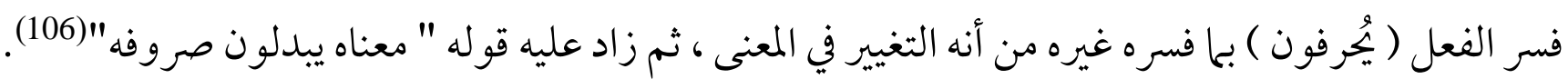

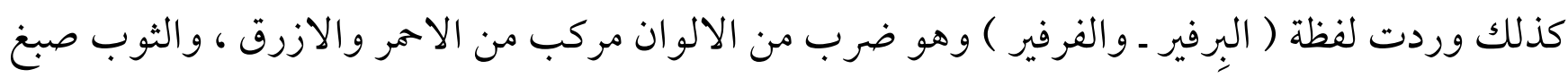

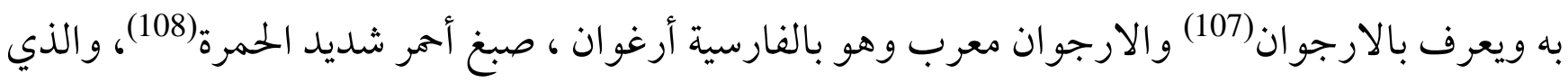

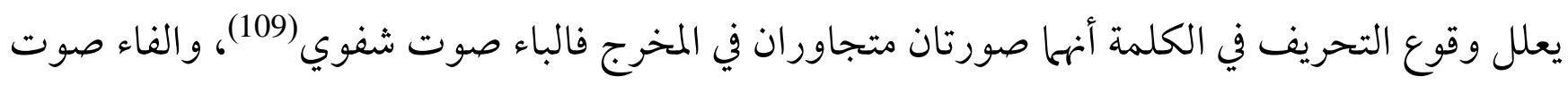
شفوي اسناني (110). إذن صو تان متقاربان في المخرج ومتحدان في الصفة ( فالباء أخت الفاء ) (111). أما التصحيف الذي ورد في معجم الالفاظ الفارسية على النحو الاتي :

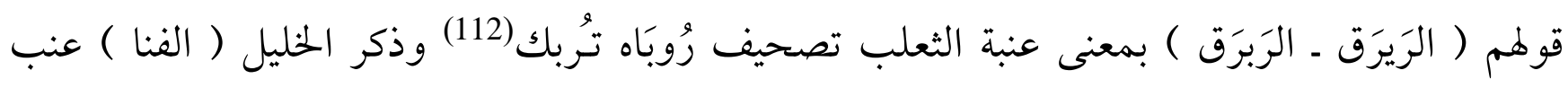

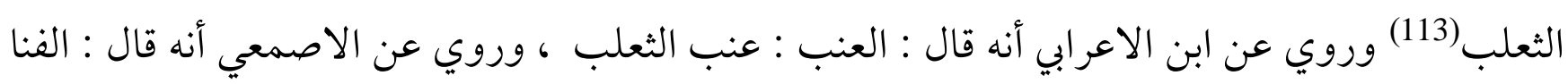
أعنب الثعلب ولم يقل عنب(114) ابن. لذا انبرى جماعة من العلماء للتصحيفات التي وقع بها الخاصة والعامة فقاموا برصدها ومتابعتها وردوها إلى

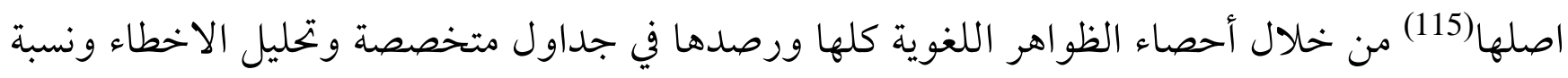
شيوعها. وكذلك قولهم ( السَدَاب ـ السّذاب ) وهو نبات يقارب شجر الرمان ورقه كالصعتر وزهره أصفر ورائحته

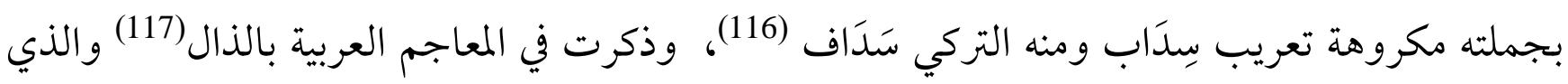

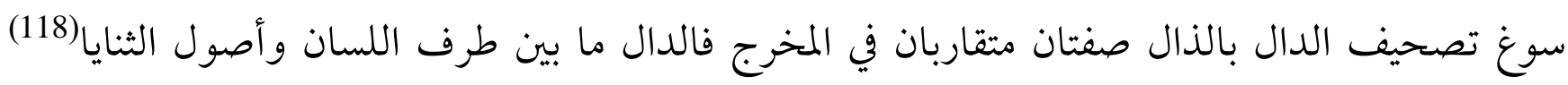
والذال ما بين طرف اللسان واطر اف الثنايا ، وكلاهما مرقق (119) وهذا ما تمتاز به العربية بكثرة التشابه بين طين

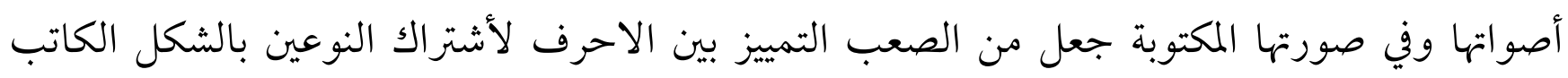

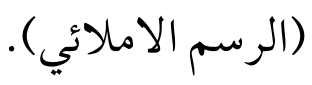


كذلك ( الحسُرو دَار ـ. الخسرو دارة ) وهي شجرة يعرف خشبها بالخو انجان وهو أقوى الأدوية في تحليل

$$
\text { الرياح تعريب خسرودارو (120). }
$$

فالذي سوغ التصحيف هنا هو الاتحاد في المخرج فالحاء والخاء هما أصوات حلقية(121) وعدها بعضهم

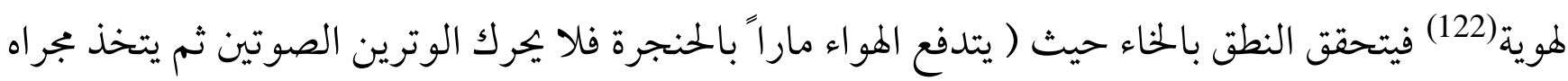

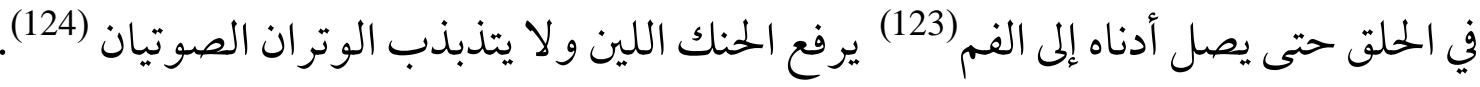
ولعل وقوع التصحف يعود إلى ظاهرة تعود تأدية الاصوات ( تفخيمها وترقيقها وتسهيلها وتخفيفها ) ما ينتج عنه خلط بين الصوت المنطوق وشكله المكتوب سواء أكان ذلك بسبب طريقة الأداء اللغوي .

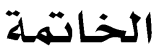

1 - أشرنا إلى أن السبب الرئيسي لـ (التدخل ) الناتج من اختلاف بين اللغتين دون إغفال لما يمكن أن يحدث من تدخل بسبب التشابه في ما بينها) ، وفي الجانب الصوتي يتو افق طردياً مع حجم الاختلاف بين اللغتين

$$
\text { من حيث ( خخارج الاصوات والعادات النطقية ـ التجمعات الصوتية ) . }
$$

2- دور العرب في وصف صفات الحروف وصفاً دقيقاً أثار دهشة المستشرقين فالعرب هم من السابقين في

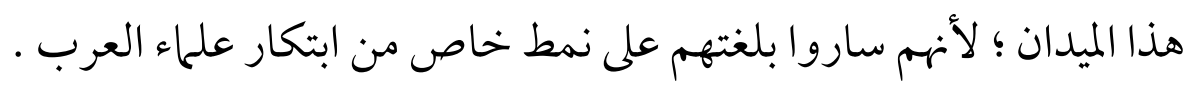

3- تفسير الظواهر الصوتية التي تعتمد على التأني وتحقيق ذوات الحروف مثل ( القلب المكاني ـ والماثلة

والمخالفة ).

4- من خلال تتبع الدراسات الصوتية ندرك أن علماء العربية كانوا على دراية بمختلف الظواهر التي تعالج

$$
\text { علم الصوت ويتجلى ذلك من خلال أعلهم المنجزة . }
$$




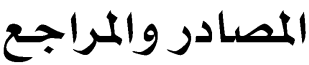

1. دُزوي:رينهارت- بيتر آن دُوزِي (المتوفى: 1300 هـ) تكملة المعاجم العربية ، نقله المى العربية وعلق عليه محمَّد سَليم

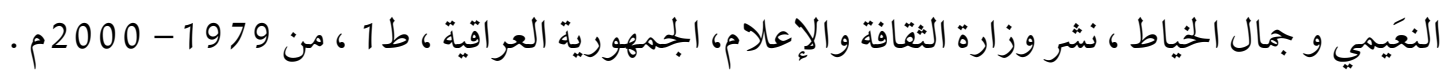

2. الجوهري : اسماعيل بن حمّاد( ت393 هـ)، الصحاح تاج اللغة وصحاح العربية تحقيق: أحمد عبد الغفور عطّار، دار

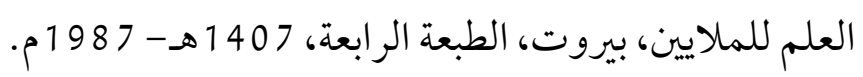

3. ابن جني :ابو الفتح بن عثمان (ت 392 هـ) الخصائص ، تحقيق محمد علي النجار ، القاهرة ،ط 2 ، 1955.

4. ابن يعيش: موفق الدين يعيش بن علي بن يعيش (ت 643 6هـ)، شرح المفصّل، المطبعة المنيرية ، مصر، (د.ت).

5. ادى شير: السيد المطران ادى شير(ت 1333هـ): معجم الالفاظ الفارسية المعربة، المطبعة الكاثولكية 1958

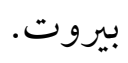

6. الازدي: أبو بكر محمد بن الحسن بن دريد الازدي (ت 321هـ) جمهرة اللغة ، تحقيق رمزي منير بعلبكي، دار العلم

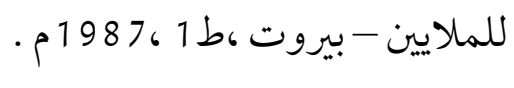

7. الاسدي : ابو البقاء يعيش بن علي بن يعيش الاسدي (643هـ) ،شرح الملوكي في التصريف ، تحقيق فخر الدين

$$
\text { قباوة ، المكتبة العربية حلب، ط1، } 1973 \text {. }
$$

8. الاستراباذي: محمد بن الحسن رضي الدين الاستراباذي ، شرح الرضي على الكافية ، تصحيح وتعليق يوسف حسن

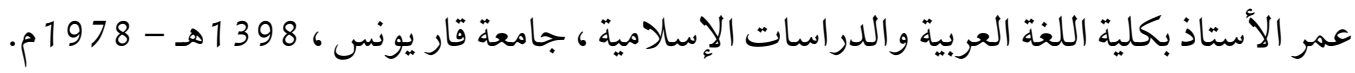
9. الانصاري : محمد بن مكرم بن علي ابو الفضل جمال الدين ابن منظور الانصاري(ت 711 7هـ) ، لسان العرب ، دار

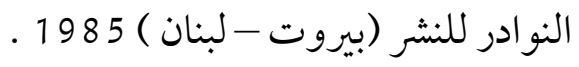

10. انيس: د. إبراهيم انيس ، في اللهجات العربية ، مطبعة الانجلو المصرية ، القاهرة 1965 . 11 1. انيس: د. إبراهيم انيس ، الأصوات اللغوية، ط5، الناشر مكتبة الانجلو المصرية ، 1975م م. 12. البكوش: الطبيب البكوش ، التصريف العربي، تحقيق صالح القرمادي ، دار النشر مؤنس، المطبعة العربية، 1992م. 13. التواب : رمضان عبد التواب ، التطور اللغوي مظاهره وعلله وقوانينه .

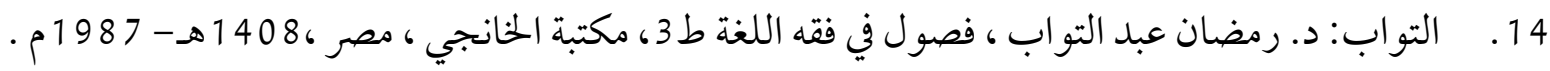
15. التواب: د. رمضان عبد التواب ، لحن العامة والتطور اللغوي، ط1 ، القاهرة ، 1967م . 
16. الجرجاني : لابي الحسن الجرجاني ( علي بن محمد بن علي المعروف بالسيد الشريف 740هـ - 1616 هـ )، التعريفات ،

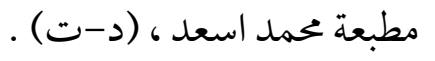

17. جني: ابي فتح عثمان بن جني ( ت 392 هـ) ، سر صناعة الاعراب، دار القلم - دمشق ، ط1 ،1985 تحقيق د.

$$
\text { حسن هنداوي · }
$$

18. جني:ابو الفتح عثمان بن جني (ت 392 هـ) ، الدراسات اللهجية والصوتية ،د حسام النعيمي ، دار الطليعة -بيروت

19 . حسن: السيد محمد بن السيد حسن (ت 869 هـ ) الراموز على الصحاح ، تحقيق علي عبد الكريم الرديني ، نشر دار

$$
\text { أسامة - دمشق ، ط } 2 \text { م } 1986 \text { م م }
$$

20 . حسن: د. علي خليف حسن ، منهج الدرس الصوتي عند العرب دار الكتب العلمية ، بيروت -لبنان ، ط1، 2011 .

1 2. الحلواني ، محمد خير الحلواني ، الو اضح في علم الصرف، دار المأمون للتراث،دمشق، 1407 هـ- 1987م.

22 2. الحموز : عبد الفتاح الحموز ، ظاهرة القلب المكاني في العربية - عللها وادلتها وتفسير انواعها ، دار عحار ( ععان -

$$
\text { الاردن )، (1986، } 198
$$

23 . الدسوقي : شيخ محمد علي الدسوقي ، خلاصة تهذيب الالفاظ العامية ، القاهرة ، 1924 ـ

24. الزبيدي : ابو بكر الزبيدي، لحن العوام، تحقيق د. رمضان عبد التواب ، القاهرة 1964 ـ

25. الزبيدي: محمد بن محمد بن عبد الرزاق الحسيني ، أبو الفيض ، الملقب بمرتضى الزبيدي (ت505 120 تاج العروس

من جواهر القاموس ،بجموعة من المحققين ، دار المداية ( ترقيم الكتاب موافق للطبع ) .

26. الزخشري أساس البلاغة : أبو القاسم محمود بن عمرو بن احمد الزخشري جار الله (ت 538 هـ) أساس البلاغة،

تحقيق احمد باسل عيون السود ، دار الكتب العلمية ، بيروت - 1998 م.

27. الزخشري: أبو القاسم محمود بن عمرو بن احمد الزخشري جار الله (ت 38 5ه) ، الفائق في غريب الحديث والاثر،

$$
\text { 28. ت تحقيق ( علي محمد البجاوي ) و ( محمد أبو الفضل إبراهيم )، دار المعرفة - لبنان ط2. }
$$

29. سيبويه الكتاب :عمرو بن عثمان بن قنبر الحارثي بالولاء والمعروف ب سيبويه (ت180هـ) الكتاب تحقيق عبد

$$
\text { السلام هارون - بيروت 1403هـ - (1403 }
$$


30. السيوطي : أبو بكر جلال الدين السيوطي( ت1119هـ)، المزهر في علوم اللغة وأنواعها شرحه محمّد جاد المولى

$$
\text { وآخران، نشر دار الجيل، بيروت، (د.ت). }
$$

1 3. شاهين: د. عبد الصبور شاهين، اثر القراءات في الأصوات والنحو العربي مكتبة الخانجي - القاهرة، ط1، 1978م.

32. العسقلاني : احمد بن علي بن محمد بن حجر العسقلاني (ت 773هـ) ، نزهة النظر في توضيح نخبة الفكر، تحقيق عبد

$$
\text { الله بن ضيف الله الرحيلي ، ط1 ، } 12001 \text {. } 200
$$

33. العسكري : ابو احمد الحسن بن عبد الله بن سعيد العسكري (ت 382 هـ ) ، تحقيق عبد العزيز احمد ، مكتبة مصطفى

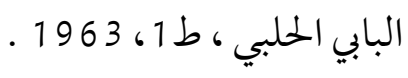

34. العسكري : ابي احمد الحسن بن عبد الله بن سعيد العسكري (ت383 هـ) ، تحقيق عبد العزيز احمد ، ط1 ، مطبعة

$$
\text { مصطفى الحلبي واو لاده - مصر } 1963 .
$$

35. العطية : خليل ابراهيم العطية ، البحث الصوتي عند العرب ، ط7، بغداد ،منشورات دار الجاحظ 1976 ـ 19.

36. عمر : احمد مختار عمر ، دراسة الصوت اللغوي، ط1، (القاهرة-مصر ) عالم الكتب 1997 .

37. عمر : د. احمد نختار عمر ، دراسة الصوت اللغوي (د. ط) ، عالم الكتب بالقاهرة (1396هـ - 1976 م ) .

38. الفراهيدي: الخليل احمد بن احد الفراهيدي (ت 170 هـ)، العين، تحقيق د. مهدي المخزومي و د. ابراهيم السامرائي، بغداد ، 1980 . 198

39. القيسي: مكي القيسي أبو محمد مكي بن أبي طالب (ت 437هـ)، مشكل إعراب القرآن، تحقيق: د. حاتم الضامن، دار الحرية للطباعة، بغداد، 1975م.

40. لتواب، د رمضان عبد التواب، المدخل الى علم اللغة ومناهج البحث اللغوي، مطبعة المدني القاهرة ، ط1 1403هـ. 1982

11 4. ما لميرنج : برتيل ما لمبرج ، علم الاصوات ، ترجمة ودراسة د. عبد الصبور شاهين ، ط1، القاهرة - مصر ، مكتبة الشباب 1984 م.

42. المبرد: ابو العباس محمد بن يزيد بن عبد الاكبر (ت 285هـ) المقتضب ، تحقيق محمد عبد الخالق عظيمة ، القاهرة، 17 1963

43. المرسي : أبو الحسن علي بن إسماعيل بن سيده المرسي (ت 458هـ) المخصص، تحقيق خليل إبراهيم جفال ، الناشر دار

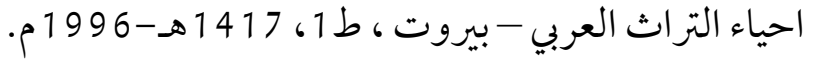


44. مطلبي: غالب فاضل مطلبي ، في الاصوات اللغوية ، جمهورية العراق - وزارة الثقافة ولاعلام ، دار الشؤون الثقافية .1984

45. الهروي: محمد بن احمد بن الازهري الهروي أبو منصور (ت 370هـ ) تهذيب اللغة، تحقيق محمد عوض مرعب ، دار

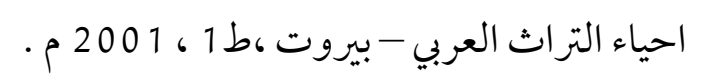

\section{الهوامش}

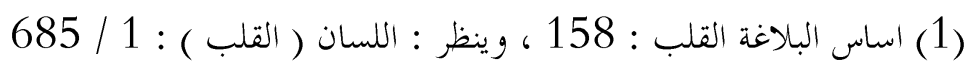

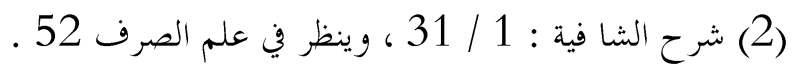

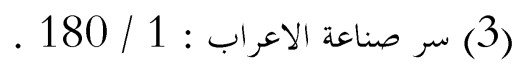

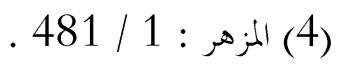

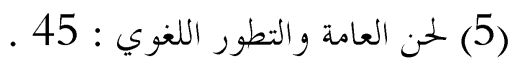

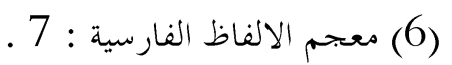
(7) (7) ابن فارس : علط الاطل

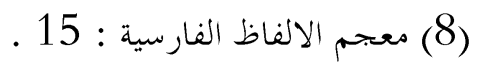

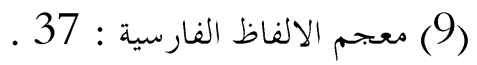

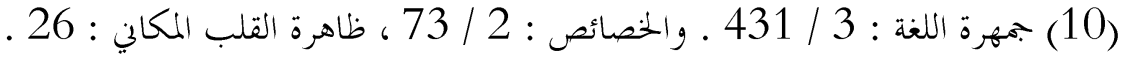

(11) معجم الالفاظ الفارسية : 149 . 14 (10) . (12) الخصائص : 35 (1)

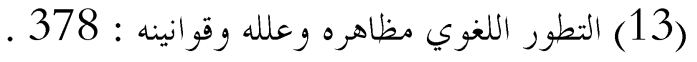

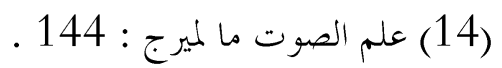

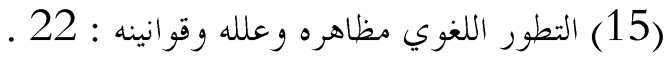

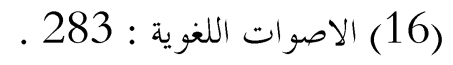

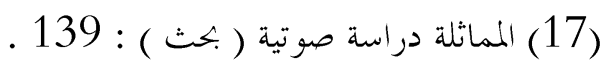

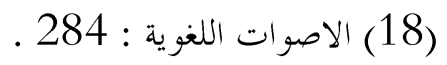

(19) معجم الالفاظ الفارسية المعربة : 111 . 11 .

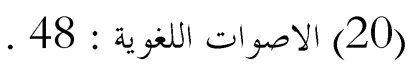

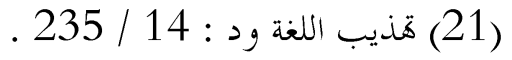


(22) المصدر نفسه : 148 / 148

(23) الالفاظ الفارسية المعربة : 111 . 142 (25) المصدر نفسه (25)

(25) الاصوات اللغوية 284 .

(26) معتم الالفاظ الفارسية : 42 (25)

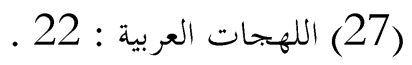

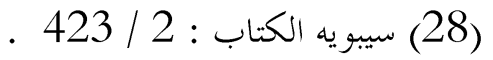

(29) معجم الالفاظ الفارسية المعربة : 73 ـ 723 (29)

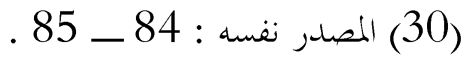

(31) المصدر نفسه : 71 (31)

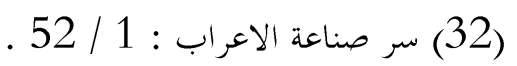

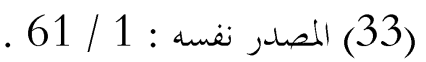

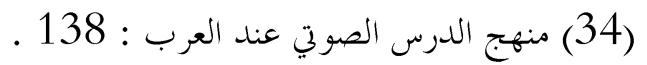

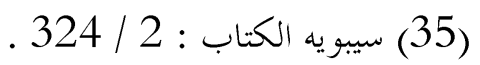

(36) معتم الالفاظ الفارسية : 108 ـ 108 (35)

(37) المصدر نفسه : 90 (38)

(38) الاصوات اللغوية : 84 ـ سر صناعة الاعراب : 1 / 60 ـ

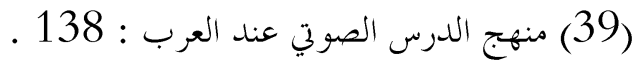

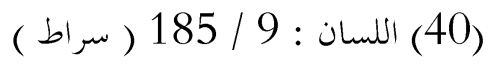

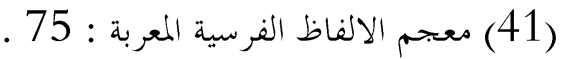

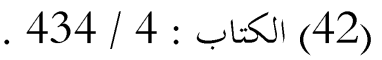

(43) هذيب الالفاظ العامية : 66 .

(44) معجم الالفاظ الفارسية المعربة : 73 .

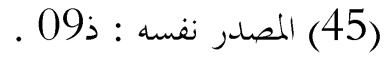

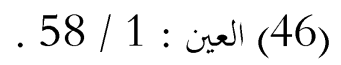

(47) الكتاب : 4 / 433 .

(48) ينظر الاصوات اللغوية : 210 .

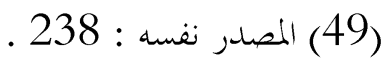

(50) شرح الملوكي في التصريف : 451 . 


$$
\begin{aligned}
& \text { (51) دراسة الصوت اللغوي : } 386 \text {. }
\end{aligned}
$$

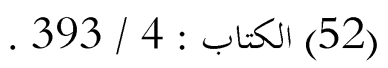

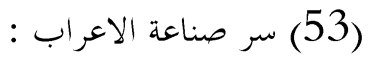

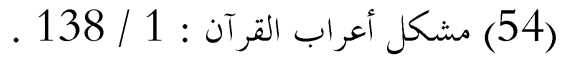

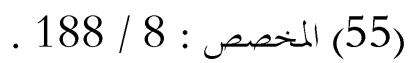

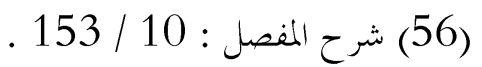

$$
\begin{aligned}
& \text { (57) التطور اللغوي مظاهره وعلله وقوانينه : } 36 \text { ـ. }
\end{aligned}
$$

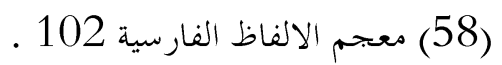

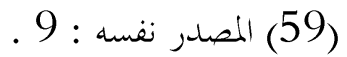

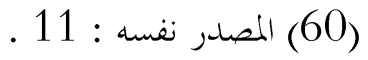

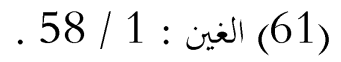

$$
\begin{aligned}
& \text { (62) المتضضب : } 1 \text { / } 1 \text { / } 246 \text {. } \\
& \text { (63) معجم الالفاظ الفارسية : } 7 \text { (65) }
\end{aligned}
$$

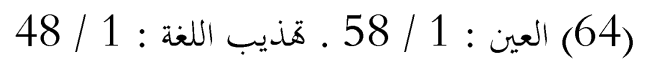

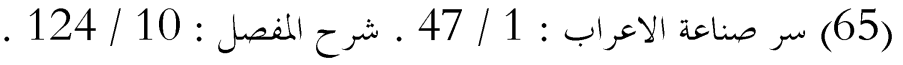

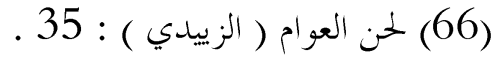

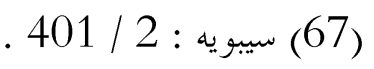

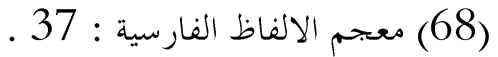

$$
\begin{aligned}
& \text { (69) المصدر نفسه : } 38 \text { (68) } \\
& \text { (70) مدخل الى علم اللغة : } 31 \text {. }
\end{aligned}
$$

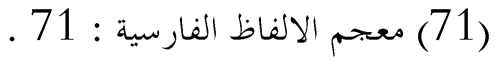

$$
\begin{aligned}
& \text { (73) المصدر نفسه : } 42 \text { (73) } \\
& 58 / 1 \text { : } 1 \text { : } 1 \text { : (73) } \\
& \text { (74) الكتاب : } 4 \text { / } 433 \text {. } 4 \text { (75) } \\
& \text { (75) الدراسات اللهجية والصوتية ( ابن جني ) : } 307 \text { ـ } 333 \text { ـ الكاب } \\
& \text { (76) الاصوات الللغوية : } 83 \text {. } \\
& \text { (77) اللسان : } 2 \text { / } 2 \text { / } 3042 \text {. }
\end{aligned}
$$

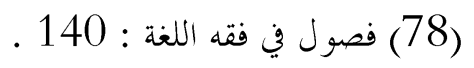

$$
\begin{aligned}
& \text { (79) العين : } 1 \text { / } 58 \text { (70) }
\end{aligned}
$$


(80)

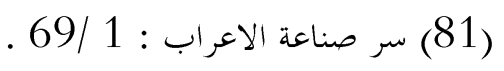

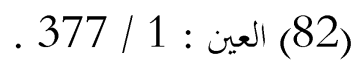

(83) هذيب اللغة : 9 / 360 ـ المخصص : 2 / / 206 ـ 206

(84) معجم الالفاظ الفارسية المعربة : 61 . 61 .

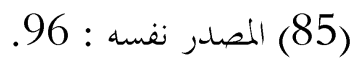

$102:==(86)$

$.110:===(87)$

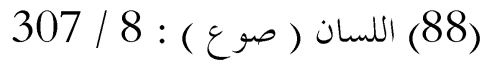

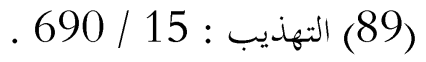

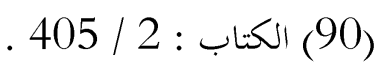

(91) شر الرضي : 1 / 26 .

(92) اثر القرات في الاصوات ( عبد الصبور شاهين ) : 377 ـ.

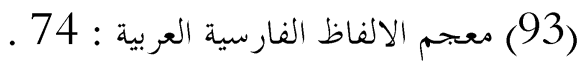

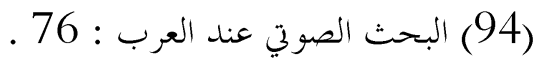

(95) معجم الالفاظ الفارسية : 38 (95)

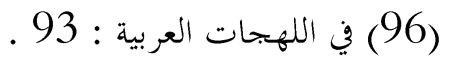

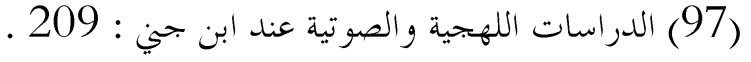

(98) معجم الالفاظ الفارسية المعربة : 38 .

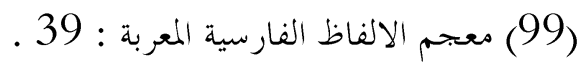

(100) التصريف العربي : 47 .

. 38 : التعريفات

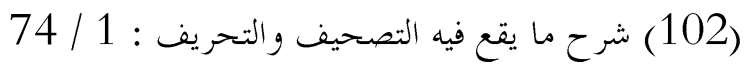

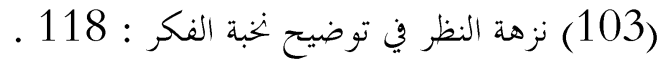

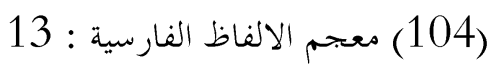

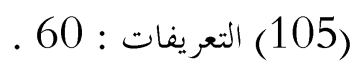

(106) تفسير القرطبي : 6 / 115 . 110

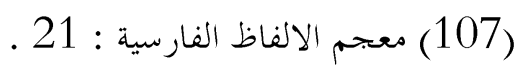

(108) الصحاح تاج اللغة : 1 / 423 ، الراموز على الصحاح : 1 / / 65 . 65 
(109) الاصوات اللغوية : 46 .

(110) الاصوات اللغوية : 47 . 40 (110)

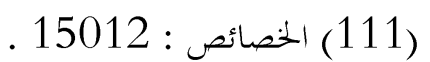

(112) معجم الالفاظ الفارسية : 71 (111)

. 37718 : 113) العين (112)

(114) مذذيب اللغة : 1 / 86 .

(115) ما يقع فيه التصحيف والتحريف

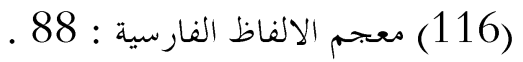

(117) الفائق في غريب الحديث : 38812 ـ

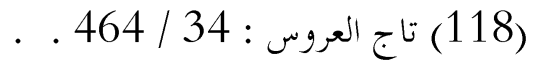

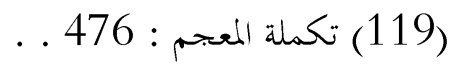

(120) معجم الالفاظ الفارسية : 50 . 50.

(121) سر صناعة الاعراب : 1 / 52.

(122) الاصوات اللغوية : 89 (123 الاصن صناعة الاعرات : 129

(123) الاصوات اللغوية : 89 . 89.

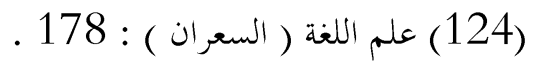

